\title{
Pulmonary sclerosing hemangioma with a rare symptom: A case report and review of the literature
}

\author{
LE ZHOU ${ }^{1}$, CHUNTANG SUN $^{2}$, YAN HUANG $^{1}$, QIAO LI ${ }^{1}$, HUAIRONG TANG $^{1}$ and YOUJUAN WANG ${ }^{1}$ \\ ${ }^{1}$ Department of Health Management Center, West China Hospital, Sichuan University; ${ }^{2}$ Department of Radiation Oncology, \\ Sichuan Cancer Hospital and Institute, Sichuan Cancer Center, School of Medicine, University \\ of Science Technology of China, Chengdu, Sichuan 610041, P.R. China
}

Received April 6, 2016; Accepted July 7, 2016

DOI: $10.3892 / \mathrm{mco} .2016 .1114$

\begin{abstract}
Pulmonary sclerosing hemangioma (PSH) is a relatively rare benign tumor. However, as it occurs only rarely, the natural course of the tumor is not well understood. In the present study, a case is presented of a 35-year-old woman who underwent intermittent fevers for more than one year. This case highlighted fever as a rare symptom of PSH. Fever is possibly one of the symptoms of PSH, although it is less likely to occur. Another symptom of the patient was that the tumor grew quickly in two months. The lesion was diagnosed as multiple sclerosing hemangioma of the lung (i.e., PSH), in which papillary, solid and sclerotic patterns appeared. Immunohistochemical evaluation of the lesion revealed positive staining for thyroid transcription factor 1 (TTF1), epithelial membrane antigen (EMA), pancytokeratin (PCK) and cytoskeleton 7 (CK7). In the present case study, the biological activity of PSH was identified to be aggressive. A review of the literature was performed in order to comment further on the clinical and pathological features of this rare disease.
\end{abstract}

\section{Introduction}

Pulmonary sclerosing hemangioma (PSH) is a relatively rare benign tumor. Due to its rarity, the symptoms and natural course of the tumor are not well understood. In the present study, a case is presented of a 35-year-old woman who underwent intermittent fevers for more than one year, and features of a rare symptom and an aggressive biological tendency are described.

Correspondence to: Dr Le Zhou, Department of Health Management Center, West China Hospital, Sichuan University, 37 Guo Xue Xiang, Chengdu, Sichuan 610041, P.R. China E-mail: 15184476542@163.com

Key words: pulmonary sclerosing hemangioma, computed tomography, pathology

\section{Case report}

A 35-year-old woman started to get fever two months prior to admission to our hospital (West China Hospital, Sichuan University, Chengdu, China). During these two months, she had intermittent fevers daily, which usually worsened during the day and improved at night, without night sweats. The highest temperature reached was $39.3^{\circ} \mathrm{C}$. Simultaneously, the patient experienced productive cough, dyspnea and right-sided chest pain. The patient had no history of shaking chills, headache, myalgia or arthralgia. The patient did not smoke or use illicit drugs. The patient had no history of exposure to cats or other animals, or exposure to risk factors for pulmonary tuberculosis or human immunodeficiency virus (HIV). Physical examination of the patient revealed entirely normal results. Routine blood and urine specimens were normal. Serological tests for syphilis and HIV were negative. Tuberculosis antibody testing and purified protein derivative (tuberculin) skin testing yielded positive results. Bacterial culture was performed, revealing that no infectious bacteria grew. Immune marker and anti-neutrophil cytoplasmic antibody testing also proved negative. Cytomegalovirus, rubella virus and herpes simplex virus immunoglobulin $\mathrm{G}$ (IgG) tests were revealed to be positive, although the patient was negative for immunoglobulin M (IgM). Inflammatory factors, including procalcitonin, serum amyloid protein, $\mathrm{C}$ - reactive protein and interleukin-6, were also negative.

A contrast-enhanced computed tomography (CT) examination of the chest on admission of the patient, performed on an Aquilion ${ }^{\mathrm{TM}} 64$ CT Scanner (Toshiba Medical Systems, Otawara, Japan), revealed innumerable lesions that varied from several millimeters to $3 \mathrm{~cm}$ in diameter in all lobes of the right lung, although none were present in the left lung. Each lesion was a round-shaped nodule with a distinct margin and homogeneous density. The CT scan revealed enlarged mediastinal and right hilar lymph nodes (Fig. 1A). In the two months following admission, the patient received anti-infection and anti-tuberculosis treatments. Clindamycin, moxifloxacin, piperacillin and tazobactam were administered, without any alleviation of the symptoms. Subsequently, the patient was administered with anti-tuberculosis treatments, including rifampicin, armazide, ethambutol and Levofloxacin, although the symptoms remained. The CT scan was subsequently re-examined. This 

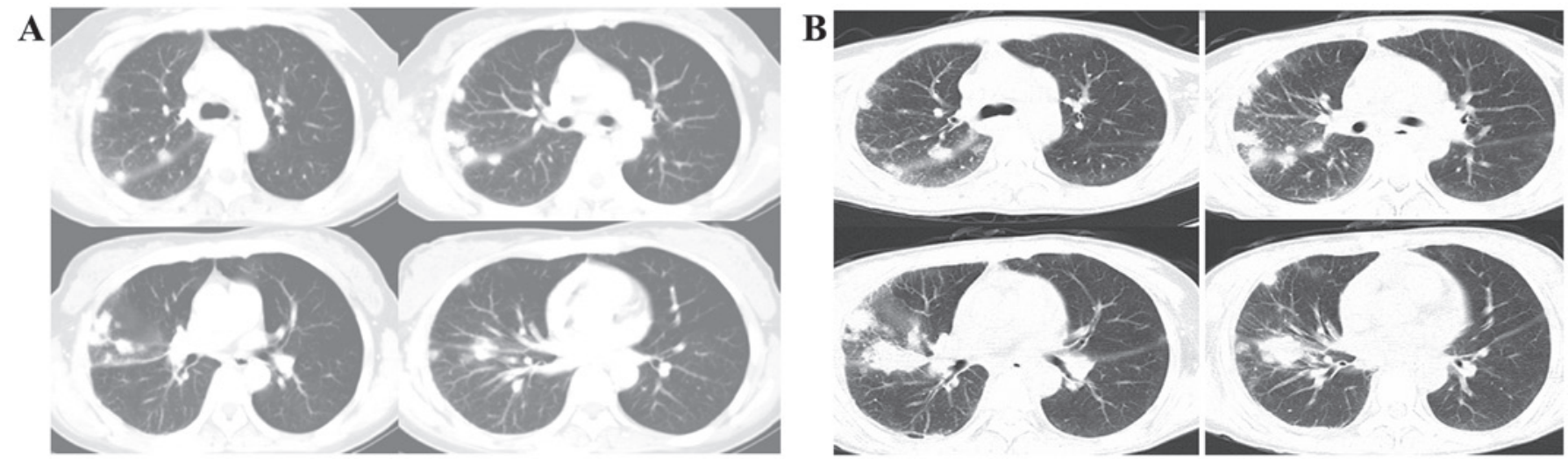

Figure 1. Comparison of the CT examinations. (A) Contrast-enhanced CT examination of the chest on admission. (B) CT examination of the patient two months later. CT, computed tomography.
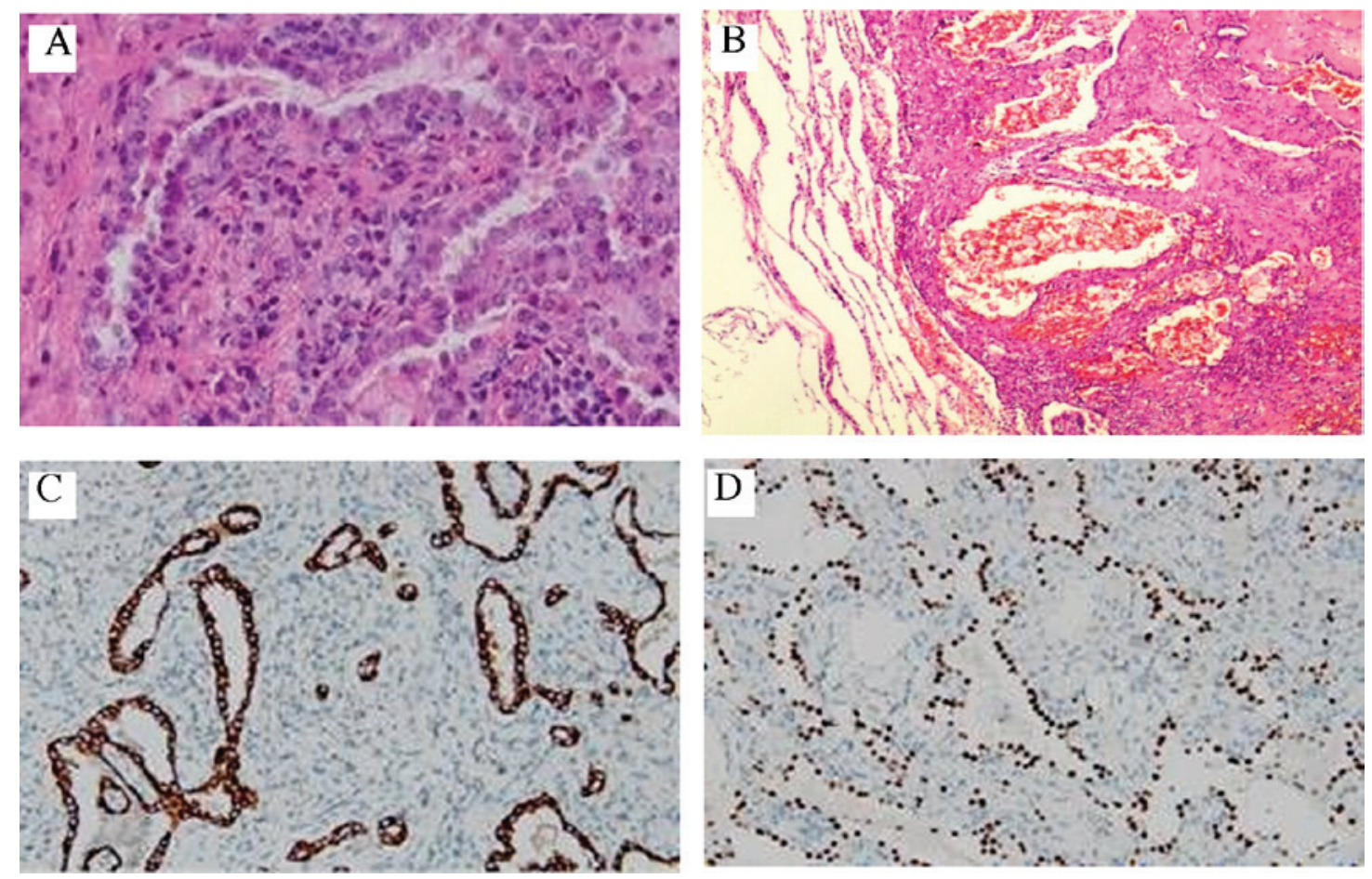

Figure 2. Pathological diagnosis of the patient. (A) The papillary pattern is illustrated (magnification, $\mathrm{x} 200$ ). Cuboidal cells are located on the papillary surface, and polygonal cells are in the stroma. (B) The sclerotic pattern is shown (magnification, $\mathrm{x} 40$ ). Hyaline collagen tissue is shown around the hemorrhagic areas. (C) Both the cuboidal and the polygonal cells stained positively for thyroid transcription factor 1 (magnification, x100). (D) Epithelial membrane antigen highlighted both the cuboidal cells and polygonal cells (magnification, $\mathrm{x} 100$ ).

revealed that the lesions had grown larger, and several lesions had coalesced. The mediastinal and right hilar lymph nodes, however, showed no further signs of increasing (Fig. 1B).

The bronchoscope and lung biopsy through percutaneous paracentesis yielded negative results. Finally, the patient underwent a thoracoscopic lung biopsy. The nodules in the right pleural cavity were biopsied. No specific bacterial infection was found in the bacterial culture of the nodules. On the basis of postoperative pathological diagnosis of paraffin-embedded tissue samples, these lesions were diagnosed as multiple sclerosing hemangiomas of the lung (i.e., as PSH), in which papillary (Fig. 2A), solid and sclerotic (Fig. 2B) patterns appeared. Immunohistochemical evaluation of the lesions revealed positive staining for thyroid transcription factor 1 (TTF1) (Fig. 2C), epithelial membrane antigen (EMA) (Fig. 2D), pancytokeratin (PCK) and cytoskeleton 7 (CK7). Staining proved to be negative for CD56, synaptophysin (Syn) and chromogranin $\mathrm{A}(\mathrm{CgA})$. The $\mathrm{Ki}-67$ labeling index was $<5 \%$.

Since the patient's general state of health was poor, she was unable to tolerate a further operation. Therefore, the patient was discharged from hospital ten days after the thoracoscopic lung biopsy. The patient did not receive any further adjuvant therapy (either chemotherapy or radiotherapy). During the follow-up for 1 year, the patient had recurring, intermittent fevers each day. The highest temperature recorded reached $39.3^{\circ} \mathrm{C}$. However, the $\mathrm{CT}$ scans did not reveal any major changes during this year. In March 2016, the patient underwent 
lobectomy of the middle lobe of the right lung in a different hospital. These lesions continued to be diagnosed as multiple sclerosing hemangiomas of the lung. Following the operation, the patient did not have a fever for one month (up to the time of writing).

\section{Discussion}

PSH is a relatively rare benign tumor, which was first described by Liebow and Hubell in 1956 (1). PSH is categorized as a 'miscellaneous tumor' according to the 2004 World Health Organization classification of lung tumors. However, as they are extremely rare manifestations, the natural course of PSHs has yet to be fully elucidated. The etiology and origin of PSH remains a controversial topic in the literature.

Concerning the clinical features, $\mathrm{PSH}$ of the lung is most frequently encountered in middle-aged women, with a peak incidence in 50-year-old women. The female to male ratio is 5:1 in this patient group (2).

Most individuals with PSH are asymptomatic, with the lesion being discovered incidentally on chest radiographs performed for other reasons, but presentations with cough, hemoptysis, chest pain, dyspnea and pleuritic pain have also been reported (3). The symptom of fever had not been mentioned previously in the literature; however, it was the predominant symptom for the patient in the present case report. This symptom cannot be explained by any of the other findings; perhaps fever may be one of the symptoms of $\mathrm{PSH}$, although it is less likely to be present.

Most PSHs arise in the peripheral parenchyma, particularly in a subpleural location. It has been reported to be more common on the right side, although cases with multiple lesions in both lungs have also been described (4). Very rarely, PSH may originate from the mediastinum (5). Devouassoux-Shishe boran et al (6) studied 100 cases of PSH which were presented, and 96 lesions (96\%) were solitary and unilateral. The tumors ranged in size from 0.3-7 cm (mean: $2.6 \mathrm{~cm}$ ). Nine PSHs (9.5\%) were $1 \mathrm{~cm}$ or smaller, whereas seven PSHs (7.4\%) were $5 \mathrm{~cm}$ or larger. The majority $(73.7 \%)$ measured $<3 \mathrm{~cm}$. PSH is almost always benign, with only $\sim 2-4 \%$ of the PSHs having nodal metastases that otherwise do not appear to affect patient prognosis (3).

The patient in the present case study has multiple nodules in the right lung. The CT scan revealed enlarged mediastinal and right hilar lymph nodes. However, it has yet to be determined whether the enlarged lymph node suggests a progressive clinical course or not. Compared with previous reports $(2,3)$, the difference was that these lesions became larger, and several lesions even coalesced in the space of two months. This case suggests that the biological behavior of PSH may become aggressive in certain cases.

The radiological features were typically non-specific. Tumors may be closely associated with the pleural surfaces, including the fissures (7). PSH is usually a solitary, well-circumscribed nodule. Calcification is seldom seen, cystic formation is rare (although it does occur), and cavitation does not occur (8). There is a typical sign in PSHs, which is termed the 'air meniscus sign': This is a lucent zone surrounding PSHs of the lung on plain films (9). This sign, particularly in a non-smoker, may be relatively specific, although it is not seen in the majority of PSHs, and it has also been reported in aspergilloma, tuberculoma, hamartoma and lung carcinoma (10).

Concerning the pathological features, macroscopically, a PSH is typically between 1 and $4 \mathrm{~cm}$, is well-defined, and is often hemorrhagic, with a variegated tan or white appearance (3). All the tumors with adjacent lung parenchyma that have been reported in the literature were well-circumscribed, although not encapsulated (5).

Microscopically, PSH is predominantly composed of two types of cells: Cuboidal surface cells that tend to differentiate into type II pneumocytes, and polygonal stromal cells that have considerable multiple differentiation potential (11). Surface cells are cuboidal and resemble reactive type II pneumocytes, whereas stromal cells are somewhat larger, with fine chromatin and inconspicuous nucleoli. PSH is histologically characterized by the presence of hemorrhagic, papillary, solid or sclerotic areas (3). The different histological patterns change abruptly from one area to another. The majority of the tumors exhibit at least three of these histological characteristics, as was also identified in the present case study. The papillae and tubular patterns were composed of uniform cuboidal cells with the morphology of bronchiolar epithelium and activated type II pneumocytes. The sclerotic pattern was composed of dense hyaline collagen tissue around the hemorrhagic areas, within the stalk of the papillae or within the solid areas. The solid pattern was composed of a sheet-like proliferation of round to polygonal cells with pale cytoplasm. In the hemorrhagic pattern, red blood cells had accumulated within cystic spaces composed of cuboidal surface cells (5).

An immunohistochemical examination revealed that the tumor cells were immunopositive for TTF1 and EMA. That TTF1 and EMA are the two epithelial markers that have been found to stain the surface cells and stromal cells of PSH most consistently in $>90 \%$ of cases not only indicates that these antibodies are the most useful for diagnostic purposes, but also provides insights into the cellular differentiation of these lesions (5). Besides TTF1 and EMA, other epithelial markers, such as PCK and CK7, were immunopositive. This provided further proof that PSH was endothelial in origin.

More recent studies $(8,11)$ have offered a variety of proposed etiologies regarding the cell line of origin, including mesothelial, mesenchymal and neuroendocrine. However, in the present case study, CD56, Syn and CgA were all negative. This eliminated the possibility of there being neuroendocrine cells within the PSH in our case. The presence of neuroendocrine cells within PSH in certain cases could represent either differentiation of primitive respiratory cells towards a neuroendocrine phenotype, as proposed by Noguchi et al (12) or it could result from a secondary neuroendocrine cell hyperplasia induced by PSH (5).

The Ki-67 nuclear antigen is associated with cell proliferation, and is detectable in the nuclei of cells undergoing cell cycling (the G1, S, G2, and M phases), although it is absent in resting ( $\mathrm{G} 0$ phase) cells. It is considered that biologically active tumors express high levels of Ki-67 nuclear antigen (13). The Ki-67 labeling index was determined to be $<5 \%$ in the present case study. These results indicate that patients with PSH do not exhibit a malignant phenotype; however, this result could not explain why the lesions became enlarged in a two-month period in our case study. Further follow-up is required to explain this phenomenon. 
In conclusion, in the present case study, a patient with the rare symptom of fever lasting for more than one year has been described. Fever may be one of the symptoms of PSH, although it is less likely to occur. Another feature of the patient was that the tumor grew quickly in two months. It may be surmised that the biological behavior of PSH could be aggressive in certain cases. Further follow-up and pathological studies will be performed on the patient.

\section{Acknowledgements}

The present study was supported by the Science Foundation of Chengdu Municipal Science and Technology Bureau (grant no. 2015-ofHM01-00254-SF).

\section{References}

1. Liebow AA and Hubbell DS: Sclerosing hemangioma (histiocytoma, xanthoma) of the lung. Cancer 9: 53-75, 1956.

2. Kuo KT, Hsu WH, Wu YC, Huang MH and Li WY: Sclerosing hemangioma of the lung: An analysis of 44 cases. J Chin Med Assoc 66: 33-38, 2003.

3. Miyagawa-Hayashino A, Tazelaar HD, Langel DJ and Colby TV: Pulmonary sclerosing hemangioma with lymph node metastases: Report of 4 cases. Arch Pathol Lab Med 127: 321-325, 2003.

4. Sugio K, Yokoyama H, Kaneko S, Ishida T and Sugimachi K: Sclerosing hemangioma of the lung: Radiographic and pathological study. Ann Thorac Surg 53: 295-300, 1992.

5. Sakamoto K, Okita M, Kumagiri H, Kawamura S, Takeuchi K and Mikami R: Sclerosing hemangioma isolated to the mediastinum. Ann Thorac Surg 75: 1021-1023, 2003.
6. Devouassoux-Shisheboran M, Hayashi T, Linnoila RI, Koss MN and Travis WD: A clinicopathologic study of 100 cases of pulmonary sclerosing hemangioma with immunohistochemical studies: TTF-1 is expressed in both round and surface cells, suggesting an origin from primitive respiratory epithelium. Am J Surg Pathol 24: 906-916, 2000.

7. Im JG, Kim WH, Han MC, Han YM, Chung JW, Ahn JM and Do YS: Sclerosing hemangiomas of the lung and interlobar fissures: CT findings. J Comput Assist Tomogr 18: 34-38, 1994.

8. Neuman J, Rosioreanu A, Schuss A, Turi G, Yung E, Trow TK, Williams L and Katz DS: Radiology-pathology conference: Sclerosing hemangioma of the lung. Clin Imaging 30: 409-412, 2006.

9. Bahk YW, Shinn KS and Choi BS: The air meniscus sign in sclerosing hemangioma of the lung. Radiology 128: 27-29, 1978.

10. Nam JE, Ryu YH, Cho SH, Lee YJ,Kim HJ,Lee DY, Choe KO and Kim SJ: Air-trapping zone surrounding sclerosing hemangioma of the lung. J Comput Assist Tomogr 26: 358-361, 2002.

11. Wang Y, Dai S and Wang E: Differential gene expressions of polygonal cells and cuboidal cells in so-called pulmonary sclerosing hemangioma. Zhongguo Fei Ai Za Zhi 10: 466-470, 2007 (In Chinese).

12. Noguchi M, Morikawa A, Kawasaki M, Matsuno Y, Yamada T, Hirohashi S, Kondo H and Shimosato Y: Small adenocarcinoma of the lung. Histologic characteristics and prognosis. Cancer 75: 2844-2852, 1995.

13. Cattoretti G, Becker MH, Key G, Duchrow M, Schlüter C, Galle J and Gerdes J: Monoclonal antibodies against recombinant parts of the Ki-67 antigen (MIB 1 and MIB 3) detect proliferating cells in microwave-processed formalin-fixed paraffin sections. J Pathol 168: 357-363, 1992. 